\title{
Using the gradient boosting decision tree (GBDT) algorithm for a train delay prediction model considering the delay propagation feature
}

\author{
Zhang, Y.D. ${ }^{a}{ }^{*}$, Liao, L. ${ }^{a}$, Yu, Q. ${ }^{a}$, Ma, W.G. ${ }^{a}$, Li, K.H. ${ }^{b}$ \\ aschool of Information Science and Technology, Southwest Jiaotong University, Chengdu, P.R. China



\begin{abstract}
A B S T R A C T
Accurate prediction of train delay is an important basis for the intelligent adjustment of train operation plans. This paper proposes a train delay prediction model that considers the delay propagation feature. The model consists of two parts. The first part is the extraction of delay propagation feature. The best delay classification scheme is determined through the clustering method of delay types for historical data based on the density-based spatial clustering of applications with noise algorithm (DBSCAN), and combining the best delay classification scheme and the k-nearest neighbor (KNN) algorithm to design the classification method of delay type for online data. The delay propagation factor is used to quantify the delay propagation relationship, and on this basis, the horizontal and vertical delay propagation feature are constructed. The second part is the delay prediction, which takes the train operation status feature and delay propagation feature as input feature, and use the gradient boosting decision tree (GBDT) algorithm to complete the prediction. The model was tested and simulated using the actual train operation data, and compared with random forest (RF), support vector regression (SVR) and multilayer perceptron (MLP). The results show that considering the delay propagation feature in the train delay prediction model can further improve the accuracy of train delay prediction. The delay prediction model proposed in this paper can provide a theoretical basis for the intelligentization of railway dispatching, enabling dispatchers to control delays more reasonably, and improve the quality of railway transportation services.
\end{abstract}

\author{
ARTICLE INFO \\ Keywords: \\ Train delay prediction; \\ Actual train operation data; \\ Delay type identification; \\ Delay propagation feature extrac- \\ tion; \\ Density-based spatial clustering of \\ applications with noise (DBSCAN); \\ k-nearest neighbor (KNN); \\ Gradient boosting decision tree \\ (GBDT);
}

Random forest (RF); Support vector regression (SVR); Multilayer perceptron (MLP)

*Corresponding author: ydzhang@swjtu.edu.cn (Zhang, Y.D.)

Article history:

Received 24 July 2021

Revised 25 October 2021

Accepted 28 October 2021



\section{References}

[1] Huang, P., Peng, Q., Wen, C., Yang, Y. (2018). Random forest prediction model for Wuhan-Guangzhou HSR primary train delays recovery, Journal of the China Railway Society, Vol. 40, No. 7, 1-9.

[2] Wen, C., Li, Z., Huang, P., Tian, R., Mou, W., Li, L. (2019). Progress and perspective of data-driven train delay propagation, China Safety Science Journal, Vol. 29, No. S2, 1-9, doi: 10.16265/i.cnki.issn1003-3033.2019.S2.001.

[3] Oneto, L., Fumeo, E., Clerico, G., Canepa, R., Papa, F., Dambra, C., Mazzino, N., Anguita, D. (2018). Train delay prediction systems: A big data analytics perspective, Big Data Research, Vol. 11, 54-64, doi: 10.1016/i.bdr.2017. $\underline{05.002}$

[4] Wang, P., Zhang, Q.-P. (2019). Train delay analysis and prediction based on big data fusion, Transportation Safety and Environment, Vol. 1, No. 1, 79-88, doi: 10.1093/tse/tdy001. 
[5] Shi, R., Xu, X., Li, J., Li, Y. (2021). Prediction and analysis of train arrival delay based on XGBoost and Bayesian optimization, Applied Soft Computing, Vol. 109, Article No. 107538, doi: 10.1016/i.asoc.2021.107538.

[6] Nair, R., Hoang, T.L., Laumanns, M., Chen, B., Cogill, R., Szabó, J., Walter, T. (2019). An ensemble prediction model for train delays, Transportation Research Part C: Emerging Technologies, Vol. 104, 196-209, doi: 10.1016/i.trc. 2019.04.026.

[7] Li, Z.-C., Wen, C., Hu, R., Xu, C., Huang, P., Jiang, X. (2020). Near-term train delay prediction in the Dutch railways network, International Journal of Rail Transportation, Vol. 9, No. 6, 520-539, doi: 10.1080/23248378.2020. 1843194.

[8] Huang, P., Wen, C., Fu, L., Lessan, J., Jiang, C., Peng, Q., Xu, X. (2020). Modeling train operation as sequences: A study of delay prediction with operation and weather data, Transportation Research Part E: Logistics and Transportation Review, Vol. 141, Article No. 102022, doi: 10.1016/i.tre.2020.102022.

[9] Gao, B., Ou, D., Dong, D., Wu, Y. (2020). A data-driven two-stage prediction model for train primary-delay recovery time, International Journal of Software Engineering \& Knowledge Engineering, Vol. 30, No. 7, 921-940, doi: $10.1142 / \mathrm{S} 0218194020400124$.

[10] Tang, Y., Xu, C., Wen, C., Li, Z., Song, S. (2019). Support vector regression models for delay time predicting considering high-speed rail facility failure, China Safety Science Journal, Vol. 29, No. S2, 18-23, doi: 10.16265/ j.cnki.issn1003-3033.2019.S2.003.

[11] Zhang, Q., Chen, F., Zhang, T., Yuan, Z.M. (2019). Intelligent prediction and characteristic recognition for joint delay of high speed railway trains, Acta Automatica Sinica, Vol. 45, No. 12, 2251-2259, doi: 10.16383/j.aas. c190188.

[12] Hu, R., Xu, C., Feng, Y., Wen, C., Wang, Q. (2019). Prediction of different types of train delay of GuangzhouShenzhen high-speed railway, China Safety Science Journal, Vol. 29, No. S2, 181-186, doi: 10.16265/i.cnki.issn 1003-3033.2019.S2.030.

[13] Zeng, Y., Chen, F., Jin, B. (2019). A prediction model for timetable delays in dispatching area using neural network, Railway Standard Design, Vol. 63, No. 3, 148-153, doi: 10.13238/j.issn.1004-2954.201812160002.

[14] Milinković, S., Marković, M., Vesković, S., Ivić, M., Pavlović, N. (2013). A fuzzy Petri net model to estimate train delays, Simulation Modelling Practice and Theory, Vol. 33, 144-157, doi: 10.1016/i.simpat.2012.12.005.

[15] Lessan, J., Fu, L., Wen, C. (2019). A hybrid Bayesian network model for predicting delays in train operations, Computers \& Industrial Engineering, Vol. 127, 1214-1222, doi: 10.1016/j.cie.2018.03.017.

[16] Pullagura, L., Katiravan, J. (2019). Train delay prediction using machine learning, International Journal of Engineering and Advanced Technology (IJEAT), Vol. 9, No. 2, 1312-1315, doi: 10.35940/ijeat.A2088.129219.

[17] Huang, P., Wen, C., Fu, L., Peng, Q., Tang, Y. (2020). A deep learning approach for multi-attribute data: A study of train delay prediction in railway systems, Information Sciences, Vol. 516, 234-253, doi: 10.1016/j.ins.2019. $\underline{12.053}$.

[18] Hansen, I.A., Goverde, R.M.P., van der Meer, D.J. (2010). Online train delay recognition and running time prediction, In: Proceedings of $1^{\text {th }}$ International IEEE Conference on Intelligent Transportation Systems, Funchal, Portugal, 1783-1788, doi: 10.1109/ITSC.2010.5625081. 
pro

\title{
Uporaba algoritma odločitvenega drevesa za povečanje gradienta (GBDT) za model napovedovanja zamude vlaka ob upoštevanju funkcije napredovanja zamude
}

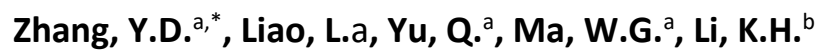 \\ aSchool of Information Science and Technology, Southwest Jiaotong University, Chengdu, P.R. China \\ bSchool of management, Xihua University, Chengdu, P.R. China
}

\begin{abstract}
POVZETEK
Natančna napoved zamude vlaka je pomembna osnova za pametno prilagajanje voznega reda vlaka. Ta članek predlaga model napovedovanja zamude vlaka, ki upošteva funkcijo napredovanja zamude. Model je sestavljen iz dveh delov. Prvi del ovrednoti funkcijo napredovanja zamude. Za oblikovanje razvrstitvene metode za razvrščanje tipov zamud za trenutne podatke je določena razvrstitvena shema $\mathrm{z}$ združitvijo metode združevanja $v$ gruče na podlagi zgodovinskih podatkov različnih tipov zamud, ki temelji na algoritmu prostorskega gručenja s šumom na podlagi gostote (DBSCAN), in z algoritma razvrščanja najbližjih sosedov (KNN). Funkcija napredovanja zamude se uporablja za količinsko opredelitev razmerja napredovanja zamude, na tej osnovi pa sta ovrednoteni funkciji napredovanje horizontalne in vertikalne zamude. Drugi del je predvidevanje zamude, ki kot vhodno funkcijo uporabi funkcijo obratovanja vlaka in funkcijo napredovanja zamude, za napoved pa uporabi algoritem odločitvenega drevesa za povečanje gradienta (GBDT). Model je bil testiran in simuliran $\mathrm{z}$ uporabo dejanskih podatkov o obratovanju vlaka ter primerjan $\mathrm{z}$ algoritmi naključni gozd (RF), regresijo podpornih vektorjev (SVR) in večplastnim perceptron (MLP). Rezultati kažejo, da lahko upoštevanje funkcije napredovanja zamude $\mathrm{v}$ modelu napovedi zamude vlaka dodatno izboljša natančnost napovedi zamude vlaka. Model napovedovanja zamud, ki ga predlagamo $\mathrm{v}$ tem članku, lahko zagotovi teoretično osnovo za pametno železniško vodenje, kar dispečerjem omogoča razumnejši nadzor nad zamudami in izboljša kakovost storitev železniškega prevoza.
\end{abstract}

\section{PODATKI O ČLANKU}

Ključne besede:

Napoved zamude vlaka;

Dejanski podatki o obratovanju vlaka;

Identifikacija vrste zamude; Ovrednotenje napredovanja zamude;

Prostorsko gručenje s šumom na podlagi gostote (DBSCAN);

k-najbližji sosed (KNN);

Odločitveno drevo za povečanje gradienta (GBDT);

Naključni gozd (RF);

Regresija podpornih vektorjev (SVR);

Večplastni perceptron (MLP)

*Kontaktna oseba:

ydzhang@swjtu.edu.cn

(Zhang, Y.D.)

Zgodovina članka:

Prejet 24. julija 2021

Popravljen 25. oktobra 2021

Sprejet 28. oktobra 2021

(i)

Content from this work may be used under the terms of the Creative Commons Attribution 4.0 International Licence (CC BY 4.0). Any further distribution of this work must maintain attribution to the author(s) and the title of 